\title{
NMR spectroscopy of hydrogen deuteride and magnetic moments of deuteron and triton
}

\author{
Yurii I. Neronov ${ }^{a}$ and Savely G. Karshenboim ${ }^{\text {b,c }}$ \\ a St. Petersburg State Institute of Fine Mechanics and Optics, St. Petersburg, \\ Sablinskaya ul. 14, St.Petersburg 197101, Russia \\ ${ }^{\mathrm{b}}$ D. I. Mendeleev Institute for Metrology (VNIIM), St. Petersburg 198005, Russia \\ ${ }^{\mathrm{c}}$ Max-Planck-Institut für Quantenoptik, 85748 Garching, Germany
}

\begin{abstract}
Magnetic moments of free and bound deuteron and triton are considered and new results for their magnetic moments (in units of that of the proton) and their $g$ factors are presented. We report on a measurement with medium-pressure hydrogen deuteride (HD) at $10 \mathrm{~atm}$, which is to be compared with the previous measurement done at $100 \mathrm{~atm}$. We confirm that the high pressure used in former experiments caused no systematic effects at a level of $10 \mathrm{ppb}$. We also reexamined a theoretical uncertainty related to screening effects in HD and HT molecules and found that previously it was underestimated. The medium-pressure result obtained for the free deuteron $\mu_{d} / \mu_{p}=0.3070122065(28)$ with a fractional uncertainty of $9.1 \cdot 10^{-9}$ is free of systematic effects related to former high-pressure experiments. The reevaluated result for triton is $\mu_{t} / \mu_{p}=1.066639908(10)$ with a fractional uncertainty of 9.3 . $10^{-9}$.
\end{abstract}

Key words: Nuclear magnetic moments, NMR, Quantum electrodynamics, $g$ factor PACS: 12.20.Ds, 21.20.Ky, 27.10, 31.30.Gs.+h, 76.60.Cq, 82.56.Hg

Magnetic moments of light nuclei have been accurately studied for quite a while. Their studies play an important role since they contribute to several high-precision measurements. First, to determine a magnetic moment of a given object (particle, nucleus etc.) via a measurement of the spin precession frequency, one has to use some known magnetic moment and light nuclei supply us with such data (see e.g. the compilation $[1,2,3]$ ). Second, to determine the fine structure constant $\alpha$, one can measure a gyromagnetic ratio of some nucleus with a value of the magnetic moment known in units of the nuclear magneton or Bohr magneton (see e.g. original results on the gyromagnetic

Email address: sek@mpq.mpg.de (Savely G. Karshenboim). 
ratio of proton [4], helion [5] and reviews [6,7] for detail). Recently magnetic moments of light nuclei $(A \leq 3)$ were in part revisited [8] to take into account higher-order binding effects and some new experimental data related to the nuclear magneton value. Here we continue our reexamination of existing data and present a new measurement of the deuteron magnetic moment.

Studies of the deuteron magnetic moment are of particular interest since this is the simplest nucleus which consists of more than one nucleon. The most accurate results on the magnetic moment of the proton [9] and deuteron [10] adopted in [7] and recommended by CODATA are based on MIT experiments with hydrogen (or deuterium) atoms with a maser. The energy levels studied were split by the magnetic field. Because of the significance of the MIT results, an independent determination of the ratio of proton-to-deuteron magnetic moments is of crucial importance. The magnetic moment of deuteron (proton) can be determined in one of the following ways:

- from the splitting of the $1 s$ sublevels in deuterium (hydrogen) as it was done at MIT with an uncertainty at a level of a part in $10^{8}[9,10]$;

- by measuring a spin precession frequency of a free deuteron (proton) as it was done for a proton with an uncertainty not better than a part in $10^{7}$ for a number of cases (mainly to calibrate the magnetic field while measuring magnetic moments of other objects);

- by studying a spin precession frequency related to proton and deuteron for a HD molecule as we discuss in detail here.

The magnetic resonance spectroscopy of $\mathrm{H}_{2}, \mathrm{D}_{2}$ and $\mathrm{HD}$ to determine the magnetic moments of proton and deuteron as well as the deuteron electric quadrupole moment was first applied in [11] about sixty years ago. Later, important advantages of the application of a HD molecule for determination of $\mu_{p} / \mu_{d}$ were pointed out by Ramsey [12]. A description of the NMR spectrum of HD can be found in detail in e.g. [13]. The results of early experiments performed about fifty years ago are discussed in [14]. Since that time the accuracy has been substantially improved and is now claimed to be at a level of a few ppb. However, the most accurate molecular experiments on HD $[15,16,17]$ and HT [18] have not been considered in Ref. [8] following the statement of [7] on their unclear uncertainty, while they were used in other compilations $[1,2,3,6]$. The earlier molecular experiments (see e.g. [19]) are less accurate and are not competitive.

We believe that potentially the quoted experiments can provide us with the most accurate data and intend to clarify their uncertainty. Here we reconsider theoretical and experimental uncertainties related to the NMR spectroscopy of molecular hydrogen and its isotopic modifications and report on the new experimental result for hydrogen deuteride. A crucial level of accuracy is a part in $10^{8}$ which is needed to verify the results of the MIT experiments $[9,10]$ 
and our goal is to reach at least this level.

In NMR experiments on molecular hydrogen and its isotopes performed in 1975-1989 [15,16,17,18] several quantities were determined with an extremely high accuracy: $\mu_{p}\left(\mathrm{H}_{2}\right) / \mu_{p}(\mathrm{HD}), \mu_{d}(\mathrm{HD}) / \mu_{d}\left(\mathrm{D}_{2}\right), \mu_{d}(\mathrm{HD}) / \mu_{p}(\mathrm{HD}), \mu_{t}(\mathrm{HT}) / \mu_{p}(\mathrm{HT})$. The claimed uncertainty was at a level of a few parts in $10^{9}$. A signal related to $\mu_{p}\left(\mathrm{H}_{2}\right)$ in the tritium experiment [18] was also observed, however, the value of $\mu_{p}\left(\mathrm{H}_{2}\right) / \mu_{p}(\mathrm{HT})$ was not determined since the pure hydrogen signal was subtracted and the data are not available any longer.

The experiments $[16,17,18]$ were performed at a high pressure of approximately $10^{7} \mathrm{~Pa}(100 \mathrm{~atm})$ and often with a sample rotation. The purpose of this paper is to check whether rotation and high pressure affect the results. The experiment reported here is performed on $\mathrm{HD}$ and $\mathrm{D}_{2}$ molecules at a pressure of $10^{6} \mathrm{~Pa}(10 \mathrm{~atm})$ without a sample rotation. We also reexamine a theoretical uncertainty appearing due to the interpretation of the proton-to-deuteron ratio at molecular HD in terms of those for free nuclei.

The most important results of the high-pressure experiments $[15,16,17,18]$ are summarized in Table 1, which contains all reliable data. The ratio of nuclear magnetic moments is easily related to measured NMR frequencies

$$
\frac{\mu_{A}}{\mu_{B}}=\frac{f_{A}}{f_{B}} \frac{I_{A}}{I_{B}},
$$

where $\mu$ is the magnetic moment, $I$ is the nuclear spin and $f$ is the NMR frequency. A value of the magnetic moment there is a bound one

$$
\mu(\text { bound })=\mu(\text { free }) \cdot(1-\sigma)
$$

The uncertainty in the calculation of the screening constant $\sigma$ are discussed separately below. A value of the NMR frequency $f$ is the unweighted average over the multiplet: a triplet for proton in HD, a doublet for deuteron in HD, triton and proton in $\mathrm{HT}$, a singlet for $\mathrm{H}_{2}$ and $\mathrm{D}_{2}$. The separation between the lines within a multiplet is determined by the constant $J$ related to the nuclear spin-spin interaction (see e.g. [13]).

The measurements were taken at room temperature. The influence of the rotational and vibrational structure on the screening constants was studied at room temperature in [16].

During the experiment described in [15] the result on $\mu_{d} / \mu_{p}$ was also derived, however, later it was found to be contradicting to MIT data for a free proton 
Table 1

Results of the high pressure experiments. Unless otherwise stated a sample was rotated. The characteristic rotation frequency $\omega_{\text {rot }}=5-10 \mathrm{~Hz}$ and the rotation axis was perpendicular to the magnetic field.

\begin{tabular}{llllll}
\hline Quantity & Value & $\begin{array}{l}\text { Magnetic } \\
\text { field, } \mathrm{T}\end{array}$ & $\begin{array}{l}\text { Pressure, } \\
\text { atm }\end{array}$ & Comment & Ref. \\
\hline$\mu_{p}(\mathrm{HD}) / \mu_{p}\left(\mathrm{H}_{2}\right)$ & $1-35.9(2) \times 10^{-9}$ & 1.52 & 130 & & {$[15]$} \\
$\mu_{d}\left(\mathrm{D}_{2}\right) / \mu_{d}(\mathrm{HD})$ & $1-42(2) \times 10^{-9}$ & 1.52 & 130 & & {$[15]$} \\
$J(\mathrm{HD})$ & $43.115(12) \mathrm{Hz}$ & 1.52 & 130 & & {$[15]$} \\
$\mu_{t}(\mathrm{HT}) / \mu_{p}(\mathrm{HT})$ & $1.066639887(3)$ & 1.49 & 100 & No rotation & {$[18]$} \\
$J(\mathrm{HT})$ & $299.3(2) \mathrm{Hz}$ & 1.49 & 100 & & {$[18]$} \\
$\mu_{h}\left({ }^{3} \mathrm{He}\right) / \mu_{p}(\mathrm{H})$ & $0.761786637(13)$ & 1.49 & 60 & & {$[16]$} \\
$\mu_{h}\left({ }^{3} \mathrm{He}\right) / \mu_{p}(\mathrm{HD})$ & $0.761786661(5)$ & 1.49 & 78 & & {$[16]$} \\
$\mu_{h}\left({ }^{3} \mathrm{He}\right) / \mu_{d}(\mathrm{HD})$ & $2.481291125(3)$ & 1.49 & 78 & & {$[16]$} \\
$\mu_{p}(\mathrm{HD}) / \mu_{d}(\mathrm{HD})$ & $3.257199655(45)$ & 1.49 & 78 & Mixture with He & {$[16]$} \\
$\mu_{p}(\mathrm{HD}) / \mu_{d}(\mathrm{HD})$ & $3.257199515(40)$ & 1.41 & 130 & & {$[17]$} \\
$\mu_{p}(\mathrm{HD}) / \mu_{d}(\mathrm{HD})$ & $3.257199496(20)$ & 4.70 & 100 & No rotation & {$[17]$} \\
$\mu_{p}(\mathrm{HD}) / \mu_{d}(\mathrm{HD})$ & $3.257199514(4)$ & 4.70 & 100 & & {$[17]$} \\
\hline
\end{tabular}

and deuteron $[9,10]$ (see $[6,7]$ for detail)

$$
\frac{\mu_{d}}{\mu_{p}}=0.307012207(5)
$$

which has a fractional uncertainty of $1.5 \cdot 10^{-8}$. A search for systematic effects led to understanding that commonly used techniques of separated inductance coils for proton and deuteron resonance provide the excitation of proton and deuteron transitions in slightly different areas and due to an inhomogeneity of the magnetic field Eq. (2) is not valid any longer and the ratio of two NMR frequencies is slightly different from the ratio of the corresponding magnetic moments. The systematic effect was explained in the last paper of series [17], however, a technique with a use of the same inductance for both resonances was already applied in the experiments with triton [18] and helion [16]. However, even after fixing this systematic source, some other questions remain to be clarified. In particular, one has to check effects due to a sample rotation, high pressure and uncertainty of theory.

Effects of the rotation were studied theoretically by Rabi, Ramsey and Schwinger in [20] (see also [14]) who found that a particle in the rotated frame feels an effective magnetic field different from that in the rest frame. The value of the 
difference depends on a gyromagnetic ratio of the particle and thus different particles see a somewhat different effective field, so that Eq. (1) is not valid any longer. The ratio of the spin precession frequencies is different from that containing the magnetic moments only. The effective correction to the field is additive and proportional to the rotation frequency, which in the former high-pressure experiments $[15,16,17]$ was perpendicular to the magnetic field. Hence the linear effect in $\omega_{\text {rot }} / \omega_{\text {NMR }}$ vanishes as long as the rotation axis is perpendicular to the magnetic field and only quadratic effects, which are negligible, are present. However, a linear effect in $\omega_{\text {rot }} / \omega_{\text {NMR }}$ of the reduced value could appear if the field and the axis are not exactly perpendicular. The rotation could also cause some mechanical and chemical problems. One of the quoted high-pressure experiments [17] observed no shift of $\mu_{p}(\mathrm{HD}) / \mu_{d}(\mathrm{HD})$ due to the rotation at a level of $6 \cdot 10^{-9}$. In our experiment we also do not apply any rotation.

To check the pressure dependence, we performed a medium-pressure experiment at 10 atm results of which are reported here. This is approximately tenfold reduction compared to previous high-pressure experiments done at a pressure from 60 to $130 \mathrm{~atm}$, with the most accurate results obtained at the highest applied pressure. A higher pressure as well as rotation served well to reduce a line width of NMR signals and in former high-pressure experiments there was no overlap between lines within multiplets (a triplet for proton and a doublet for deuteron in HD with a deuteron singlet from $\mathrm{D}_{2}$ ). In the present experiment the lines within multiplets are overlapping.

Let us briefly describe our experiment. We used a commercial NMR spectrometer Bruker CXP 300 with a superconductive magnet producing the magnetic field of 7.05 T. The sample consisted of an external tube (\#508-UP by Norell, Inc.) and an internal capillary. The internal capillary was filled with gas of $\mathrm{HD}$ and $\mathrm{D}_{2}$ at a pressure of $10 \mathrm{~atm}$. The signal of the gas sample is relatively weak and we need some time to collect reliable data. In our experiment the measurement time was one hour. To simplify a calibration procedure, we filled the external tube with acetone $\left(\mathrm{CH}_{3}\right)_{2} \mathrm{CO}$ and deutero-acetone $\left(\mathrm{CD}_{3}\right)_{2} \mathrm{CO}$.

Acetone provides strong and narrow lines and we controlled the magnetic field by fixing the position of the line of deutero-acetone

$$
f_{d}\left(\left(\mathrm{CD}_{3}\right)_{2} \mathrm{CO}\right)=46072631.58(1) \mathrm{Hz}
$$

and measured the acetone signal

$$
f_{p}\left(\left(\mathrm{CH}_{3}\right)_{2} \mathrm{CO}\right)=300135552.4(1) \mathrm{Hz}
$$

At the next step we studied the proton triplet lines (see Fig. 1) shifted from 


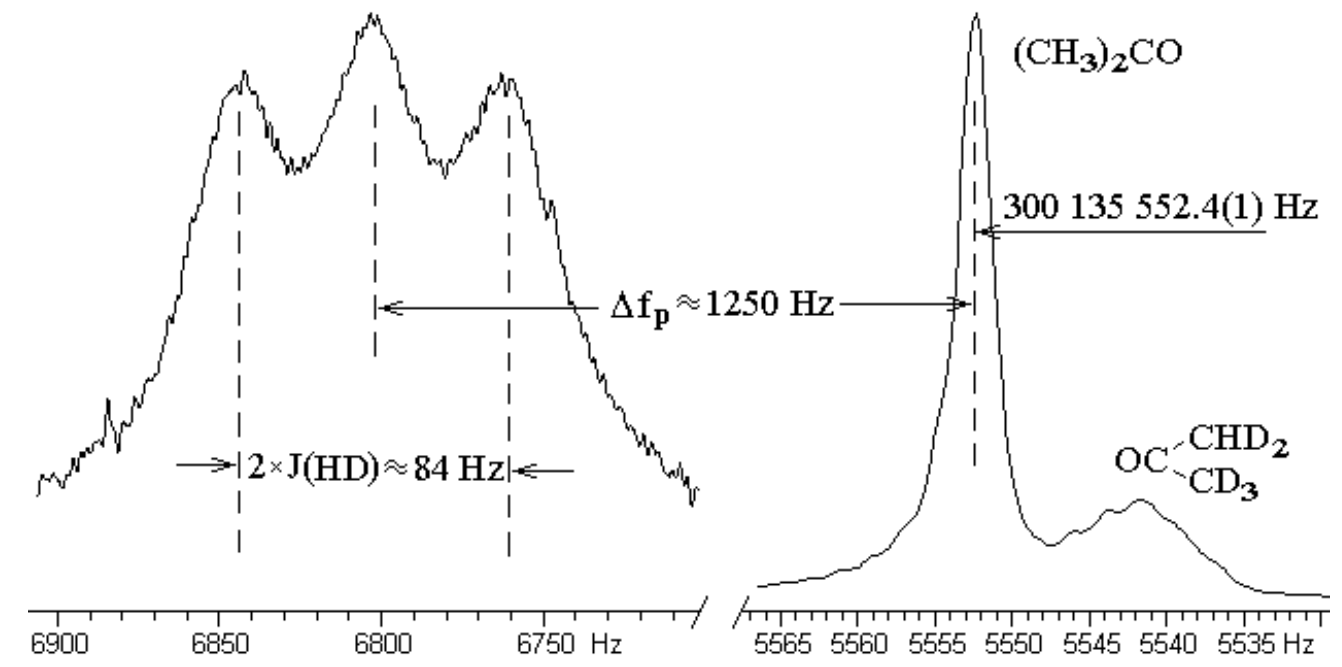

Fig. 1. The proton NMR spectrum: acetone lines and HD triplet.

the acetone line by

$$
\Delta f_{p}=f_{p}(\mathrm{HD})-f_{p}\left(\left(\mathrm{CH}_{3}\right)_{2} \mathrm{CO}\right)=1250.3(5) \mathrm{Hz}
$$

with the line separation within the triplet related to the hyperfine nuclear spin-spin constant $J(\mathrm{HD})$ and discussed below.

The other measurement was related to the deuteron lines (see Fig. 2). We determined the NMR frequency of the deuteron HD doublet shifted from the deutero-acetone line by

$$
\Delta f_{d}=f_{d}(\mathrm{HD})-f_{d}\left(\left(\mathrm{CD}_{3}\right)_{2} \mathrm{CO}\right)=198.8(4) \mathrm{Hz}
$$

with the line separation within the doublet determined by $J(\mathrm{HD})$, and the deuteron singlet line from $\mathrm{D}_{2}$ slightly shifted with respect to the center of the deuteron doublet by approximately $2 \mathrm{~Hz}$.

With these frequencies measured we arrive to a result

$$
\begin{aligned}
\frac{\mu_{p}(\mathrm{HD})}{\mu_{d}(\mathrm{HD})} & =\frac{1}{2} \frac{f_{p}\left(\left(\mathrm{CH}_{3}\right)_{2} \mathrm{CO}\right)+\Delta f_{p}}{f_{d}\left(\left(\mathrm{CD}_{3}\right)_{2} \mathrm{CO}\right)+\Delta f_{d}} \\
& =3.25719949(3)
\end{aligned}
$$

with a fractional uncertainty of $9 \mathrm{ppb}$. The uncertainty is mainly due to a line fitting procedure. The details will be published elsewhere [21]. We hope that by improving statistics the errors due to the fitting will be reduced. 


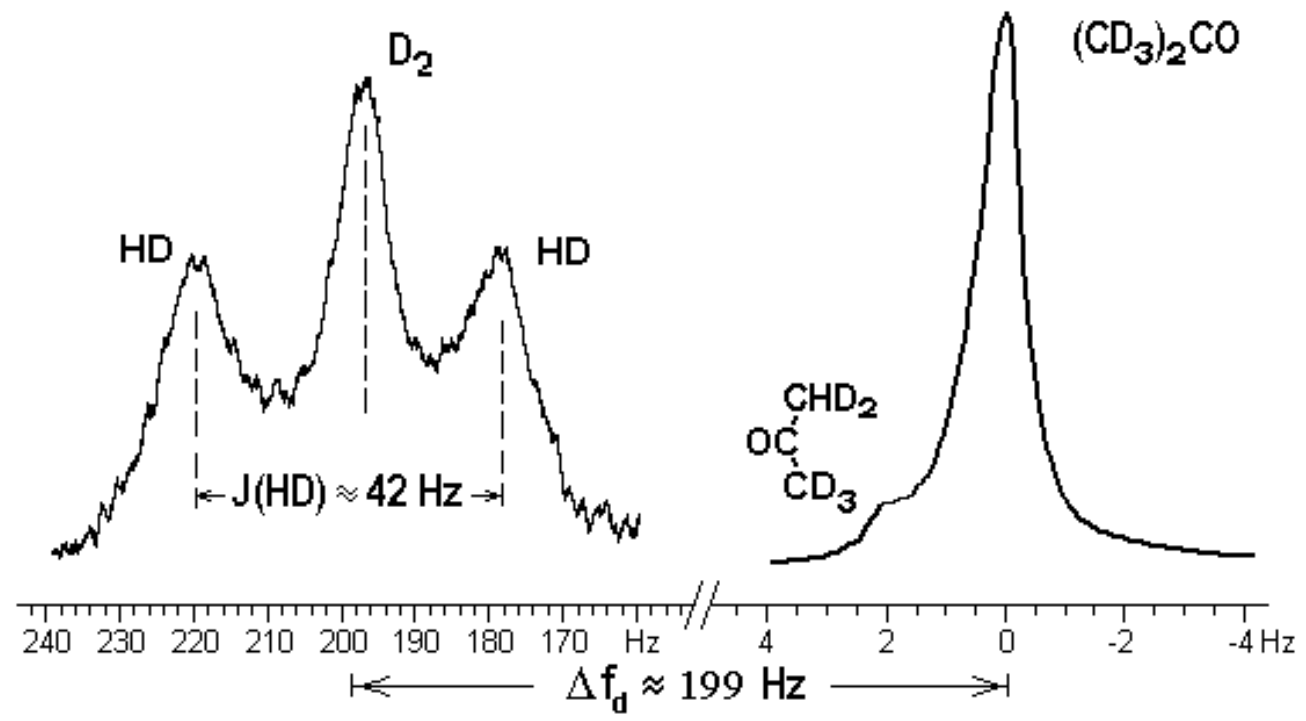

Fig. 2. The deuteron NMR spectrum: deutero-aceton lines, HD doublet and $\mathrm{D}_{2}$ singlet.

To check our results, we have performed several comparisons with previous results, which are discussed below.

Acetone. The acetone ratio $\left(f_{p} / f_{d}\right)$ is found to be in fair agreement with the data previously obtained at a lower magnetic field of $1.5 \mathrm{~T}$ and $4.7 \mathrm{~T}$ with rotation $[22,23]$. In the present experiment the acetone frequencies are related to the position of the maximum of the lines, while for an interpretation in terms of magnetic moments one has to separate the dominant proton and deuteron modes and weak contributions related to molecules with incomplete substitution of hydrogen for deuterium, $(\mathrm{CD})_{3}\left(\mathrm{CHD}_{2}\right) \mathrm{CO}$, which affect the maximum of stronger lines. Such a correction can be different under different conditions and the ratio for the quoted proton and deuteron frequencies is not exactly the same as the ratio of the proton and deuteron magnetic moments in acetone molecules. In this paper we have not considered this small correction because the acetone measurements were used for the normalization only and the interpretation of the line maximum is not important for such a purpose.

Hyperfine interaction constant $J(\mathrm{HD})$. The proton triplet should consist of three Lorentzian lines separated by the same interval as the two lines of the deuteron doublet related to the HD molecule (see Figs. 1 and 2). The separation is equal to the hyperfine constant $J(\mathrm{HD})$ (see e.g. [13]). The present results are 41.8(17) $\mathrm{Hz}$ (from the proton spectrum) and 40.8(15) $\mathrm{Hz}$ which both agree with a high-pressure result of 43.115(12) Hz [15] listed in Table 1.

Isotopic shift for deuteron. Simultaneous observation of the deuteron singlet related to $\mathrm{D}_{2}$ and the deuteron HD doublet provides us with another test (see 
Fig. 2). The result obtained is

$$
\frac{\mu_{d}\left(\mathrm{D}_{2}\right)}{\mu_{d}(\mathrm{HD})}=1-45(7) \times 10^{-9}
$$

which is less accurate but consistent with the high-pressure value of $1-42(2) \times$ $10^{-9}[15]$.

All tests showed fair agreement with high-pressure results obtained applying a sample rotation. The main result of this paper in Eq. (8) also agrees with the high-pressure values listed in Table 1. The result (8) is more reliable than the tests with the isotopic effects and the hyperfine structure. The result (8) comes from average frequencies of proton triplet and deuteron doublet which are relatively immune to line-shape effects. In contrast, differential characteristics like the hyperfine separation inside the triplet and the doublet or the isotopic shift of the center of gravity of the deuteron doublet with respect to the singlet from $\mathrm{D}_{2}$ are sensitive to such effects.

Theoretical calculations for shielding and hyperfine constants were started by Ramsey $[24,25]$. Now they are substantially improved. We follow the examination in [18], however, we pay our attention to a proper estimation of the uncertainty of the quoted calculation.

The result presented in paper [18] stated

$$
\begin{aligned}
\sigma_{t}(\mathrm{HT})-\sigma_{p}(\mathrm{HT}) & =20.4 \times 10^{-9} \\
\sigma_{d}(\mathrm{HD})-\sigma_{p}(\mathrm{HD}) & =15.0 \times 10^{-9} .
\end{aligned}
$$

This result was derived as an evaluation of some matrix elements with the wave functions related to the Born-Oppenheimer approximation. All the perturbative operators to be averaged are of the order of $\alpha^{2}\left(m_{e} / m_{p}\right)$ in atomic units.

The uncertainty of the calculation was not presented explicitly in [18] and the final results for deuteron and triton did not include any theoretical uncertainty. However, at least two sources of uncertainties were mentioned by the authors and we consider them here. Note that any detail in [18] is related to HT, while here we concentrate on HD, and thence any contribution to the HD case is multiplied by roughly 0.75 as explained in [18].

- The result [18] is a sum of eight terms. However, three of them were estimated as being smaller than $4.5 \cdot 10^{-10}$ each. An additional term discussed there was estimated to be below than $1.5 \cdot 10^{-10}$. Since no details on the three terms are presented, we consider the missed terms as correlated and 
estimate their sum as $\pm 1.5 \cdot 10^{-9}$ or approximately $10 \%$ of the value of $\sigma_{d}(\mathrm{HD})-\sigma_{p}(\mathrm{HD})$.

- The wave functions in [18] are related to the binding energy which is approximately $5 \%$ different from a real one and due to that authors stated that the uncertainty cannot be lower than $5 \%$.

There may be some other sources due to the method of the calculation in [18] which are beyond consideration in this paper. We hope to decrease the experimental uncertainty for the ratio of the magnetic moments to a few ppb and thus the theoretical calculations need to be substantially improved.

Here we estimate the uncertainty of the calculation in Ref. [18] as 15\% and thus the results (10) and (11) of [18] should be corrected to

$$
\begin{aligned}
\sigma_{t}(\mathrm{HT})-\sigma_{p}(\mathrm{HT}) & =20(3) \times 10^{-9} \\
\sigma_{d}(\mathrm{HD})-\sigma_{p}(\mathrm{HD}) & =15(2) \times 10^{-9}
\end{aligned}
$$

Due to the theoretical correction (13), the result (8) leads for a free proton and deuteron:

$$
\begin{aligned}
\frac{\mu_{p}(\text { free })}{\mu_{d}(\text { free })} & =\frac{\mu_{p}(\mathrm{HD})}{\mu_{d}(\mathrm{HD})} \times\left(1-\left[\sigma_{d}(\mathrm{HD})-\sigma_{p}(\mathrm{HD})\right]\right) \\
& =3.25719944(3)
\end{aligned}
$$

and

$$
\frac{\mu_{d}(\text { free })}{\mu_{p}(\text { free })}=0.307012210(3)
$$

with a fractional uncertainty of $9 \mathrm{ppb}$, which agrees with both high-pressure data and the maser result. We summarize results on determination of the deuteron-to-proton ratio in Fig. 3.

Mixing different gaseous substances, one can cancel systematic sources due to macroscopic screening effects, perturbations of electronic wave functions etc. and to compare a given magnetic moment with a probe one. A promising probe magnetic moment is that in molecular hydrogen, which is one of very few atomic and molecular systems where the binding corrections to the nuclear magnetic moment can be in principle calculated $a b$ initio with a high accuracy. In this way it is very important to have more experimental data. A study of isotopic and hyperfine effects is an important step towards theoretical understanding of the molecular screening effects. Meanwhile, the comparison of the proton-to-deuteron (and proton-to-triton) ratio of magnetic moments related 


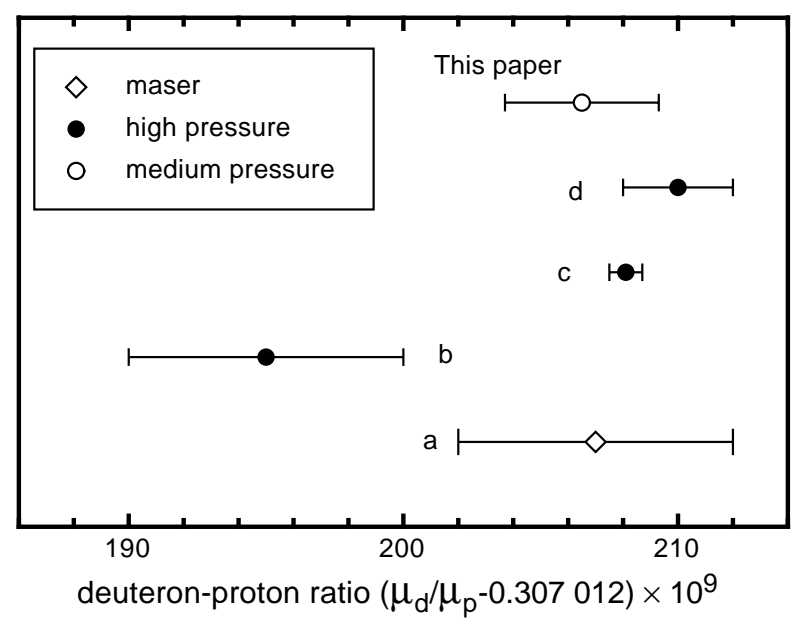

Fig. 3. The deuteron-to-proton ratio of the magnetic moments: the maser result $a$ is taken from [7], the result $b$ is related to an experiment with admixure of helium, while the results $c$ and $d$ correspond to the measurement at a pressure of $100 \mathrm{~atm}$ with/without rotation of a sample. The high-pressure results have an uncertainty corrected according to Eq. (12). See Table 1 for references and other detail.

to the same molecule allows accurate relative measurements with systematic effects essentially reduced. We have demonstrated that the high-pressure data obtained for HD molecules are reliable and confirmed them within the accuracy of our experiment. We have also carefully investigated a theoretical uncertainty which happens to be higher than a statistical uncertainty of the former high-pressure experiments $[17,18]$, but below the uncertainty of the present medium-pressure experiment.

Table 2

Final results obtained at medium-pressure. The deuteron results are achieved experimentally. The triton results are obtained adjusting the uncertainty.

\begin{tabular}{llc}
\hline Quantity & Value & Fractional uncertainty \\
\hline$\mu_{p}(\mathrm{HD}) / \mu_{d}(\mathrm{HD})$ & $3.257199531(29)$ & $8.8 \cdot 10^{-9}$ \\
$\mu_{t}(\mathrm{HT}) / \mu_{p}(\mathrm{HT})$ & $1.066639887(10)$ & $8.8 \cdot 10^{-9}$ \\
$\mu_{p}($ free $) / \mu_{d}($ free $)$ & $3.257199482(30)$ & $9.1 \cdot 10^{-9}$ \\
$\mu_{d}($ free $) / \mu_{p}($ free $)$ & $0.3070122065(28)$ & $9.1 \cdot 10^{-9}$ \\
$\mu_{t}($ free $) / \mu_{p}($ free $)$ & $1.066639908(10)$ & $9.3 \cdot 10^{-9}$ \\
$g_{d}($ free $)$ & $0.857438240(12)$ & $1.3 \cdot 10^{-8}$ \\
$g_{t}($ free $)$ & $2.97896244(4)$ & $1.4 \cdot 10^{-8}$ \\
\hline
\end{tabular}

We list in Table 2 the results for deuteron obtained in the present paper and the reexamined results for HT. We estimate the experimental HT uncertainty by the uncertainty of the present medium-pressure experiment since no discrepancy between medium-pressure and high-pressure data for HD has been 
detected. The theoretical uncertainty is taken from (12). Even with the reestimated and thus enlarged uncertainty the triton result obtained from HT is the most accurate one (cf. [26,27]) and the deuteron result has approximately an 1.5 times lower uncertainty than the maser result $(3)[9,10,7]$. We consider our experimental uncertainty here as rather somewhat overestimated and hope to improve medium-pressure measurements. We note, however, that presently the theory also substantially contributes to the uncertainty for the free magnetic ratio and further theoretical progress to obtain more reliable and more accurate data is strongly needed.

The experiment has been performed with the NMR spectrometer at the State Research Institute of Highly Pure Biopreparations (St. Petersburg) and the authors are grateful to B. P. Nikolaev and Yu. N. Tolparov for their hospitality and cooperation. The authors would like to thank Anatoly Barzakh, Jim Friar, Peter Mohr and Victor Yakhontov for useful and stimulating discussions. The work of SGK was supported in part by RFBR under grant 02-02-07027.

\section{References}

[1] P. Raghavan, At. Data. Nucl. Data Tables 42, 189 (1989).

[2] I. Mills et al., Quantities, Units and Symbols in Physical Chemistry. Blackwell Science. Ocford, 1993.

[3] R. B. Firestone, Table of Isotopes (John Wiley \& Sons, Inc., 1996).

[4] E. R. Williams, G. R. Jones, Jr., S. Ye, R. Liu, H. Sasaki, P. T. Olsen, W. D. Phillips, and H. P. Layer, IEEE Trans. Instrum. Meas. 38, 233 (1989).

[5] V. Y. Shifrin, P. G. Park, V. N. Khorev, C. H. Choi, and C. S. Kim; IEEE Trans. Instrum. Meas. 47, 638 (1998).

[6] E. R. Cohen and B. N. Taylor, Rev. Mod. Phys. bf 59, 1121 (1987).

[7] P. J. Mohr and B. N. Taylor, Rev. Mod. Phys. 72, 351 (2000).

[8] S. G. Karshenboim and V. G. Ivanov, Phys. Lett. B566, 27 (2003).

[9] P. F. Winkler, D. Kleppner, T. Myint and F. G. Walther, Phys. Rev. A 5, 83 (1972); corrected according to a private communication with D. Kleppner as quoted in [7].

[10] W. D. Phillips, D. Kleppner, and F. G. Walther, private communication (1984), quoted as in [7].

[11] J. M. B. Kellogg, I. I. Rabi, N. F. Ramsey and J. R. Zacharias, Phys. Rev. 56, 728 (1939); 57, 677 (1940).

[12] N. F. Ramsey, Phys. Rev. 85, 688 (1952). 
[13] M. Karplus and R. N. Porter. Atoms and Molecules: An Introductory For Students of Physical Chemistry. W. A. Benjamin, Inc. 1970.

[14] N. F. Ramsey. Molecular Beams. Oxford University Press, 1956.

[15] Y. I. Neronov, A. E. Barzakh, and K. Mukhamadiev, Sov. Phys. JETP 42, 950 (1975).

[16] Yu. I. Neronov and A. E. Barzakh, Sov. Phys. JETP 48, 769 (1978).

[17] M. V. Gorshkov, Y. I. Neronov, E. N. Nikolaev, Y. V. Tarbeev, and V. L. Tal'roze, Sov. Phys. Dokl. 34, 362 (1989).

[18] Y. I. Neronov and A. E. Barzakh, Sov. Phys. JETP 45, 871 (1977).

[19] T. F. Wimett, Phys. rev. 91, 499 (1953).

[20] I. I. Rabi, N. F. Ramsey and J. Schwinger, Rev. Mod. Phys. 26, 167 (1954).

[21] Yu. I. Neronov and S. G. Karshenboim, in preparation.

[22] Yu. I. Neronov, D. M. Kaminker and Kh. Mukhamadiev, Theor. Exp. Chem. 10, 161 (1974).

[23] A. A. Anselm and Yu. I. Neronov, Sov. Phys. JETP 61, 1154 (1985).

[24] N. F. Ramsey, Phys. Rev. 78, 699 (1950).

[25] N. F. Ramsey and E. M. Purcell, Phys. Rev. 85, 143(1952).

[26] W. Duffy, Jr., Phys. Rev. 115, 1012 (1959).

[27] J. M. A. Al-Rawi, J. P. Bloxsidge, C. O'Brien, D. E. Caddy, J. A. Elvidge, J. R. Jones and E. A. Evans, J. Chem. Soc. Perkin II, 1635 (1974) 